\title{
Effets du dinitro-orthocrésol (DNOC) sur la levée de dormance du pommier 'Golden Delicious' cultivé en plaine marocaine
}

Ahmed Mahhou*, Afif El Phil

Département d'Horticulture, Institut Agronomique et Vétérinaire Hassan II, BP 6202-Instituts, Rabat, Maroc

a.mahhou@iav.ac.ma
${ }^{*}$ Correspondance et tirés à part

Fruits, 2001, vol. 56, p. 367-376 (C) $2001 \mathrm{Cirad} /$ EDP Sciences All rights reserved

Resumen Español, p. 376

\section{Effects of dinitro-orthocresol (DNOC) on the dormancy breaking of 'Golden Delicious' apple trees cultivated in the Moroccan plain.}

Abstract - Introduction. The lack of winter chilling in the Moroccan plains constitutes a limiting factor for apple production. The effects of dinitro-orthocresol (DNOC), known to overcome dormancy of other fruit trees, were evaluated regarding bud break of 'Golden Delicious' apple trees grown under such climatic conditions. Materials and methods. DNOC at $0.15 \%$ combined with 3 or $5 \%$ of mineral oil was applied at the end of February or one week later, 1996. The evaluation of the treatments concerned the rates of bud break, flowering, fruit set, vegetative growth and the yield. Results. The treatments advanced and improved the rates of vegetative and floral bud break, flowering intensity, fruit see, vegetative growth as well as yield and fruit size. Conclusion. The mixture [DNOC + mineral oil] advanced and gathered bud break of both vegetative and floral buds, improved yield and fruit size and yield of 'Golden Delicious' apple trees. This mixture could thus be used to overcome the lack of winter chilling in Moroccan zones characterized by mild winters.

Morocco / Malus / dormancy breaking / dnoc / mineral oils / growth / flowering / fruiting / yields

\section{Effets du dinitro-orthocrésol (DNOC) sur la levée de dormance du pommier 'Golden Delicious' cultivé en plaine marocaine.}

Résumé - Introduction. L'insuffisance en froid hivernal dans les zones de plaine au Maroc constitue un facteur limitant à la production du pommier. Les effets du dinitro-orthocrésol (DNOC), produit connu pour lever la dormance chez d'autres arbres fruitiers, ont été évalués vis-à-vis du débourrement de pommiers 'Golden Delicious' cultivés dans de telles conditions climatiques. Matériel et méthodes. Un mélange de DNOC à $0,15 \%$ et d'huile de pétrole utilisée a $3 \%$ ou $5 \%$ a été appliqué à des pommiers, à deux dates différentes. La production des arbres ainsi traités a été comparée à celle d'arbres témoins non traités. L'évaluation des traitements a porté sur les taux de débourrement des bourgeons végétatifs et floraux, la floraison, la nouaison, la croissance végétative et la production. Résultats. La solution utilisée a avancé la date de débourrement et amélioré de façon significative les taux de débourrement des bourgeons végétatifs et floraux, la croissance végétative, ainsi que le rendement et le calibre des fruits. Conclusion. Le mélange [DNOC + huile du pétrole] a permis d'avancer et de regrouper le débourrement de l'ensemble des bourgeons et a amélioré le calibre et la production du pommier 'Golden Delicious'. Ce mélange pourrait donc être utilisé pour pallier le manque en froid hivernal des zones de plaine du Maroc, caractérisées par un hiver doux.

Maroc / Malus / levée de dormance / dnoc / huile minérale / croissance / floraison / fructification / rendement 


\section{Introduction}

Chez les espèces à feuilles caduques, la dormance, ou repos végétatif, est une forme d'adaptation et de résistance aux conditions hivernales rigoureuses des zones tempérées. Les facteurs climatiques induisent des transformations morphologiques et physiologiques qui, selon leur nature, favorisent l'instauration, le prolongement ou la levée de dormance des organes concernés.

Chez les rosacées fruitières, le froid constitue un facteur indispensable à la levée de dormance. Dans les plaines marocaines, son insuffisance limite les performances du pommier et conduit à l'apparition d'une «dormance prolongée »qui se rencontre dans les régions à hivers doux. Un manque en froid provoque un désordre physiologique de la croissance végétative et de la fructification. Cette insuffisance se manifeste par un affaiblissement général de l'arbre, un retard de débourrement, un étalement de la floraison, un faible rendement et un fruit de médiocre qualité. L'intensité de ces troubles est d'autant plus grande que le manque en froid est important et que la variété est sensible à ce facteur.

Selon Petri [1], les effets du manque en froid sur pommiers peuvent être résumés comme suit : taux de débourrement faible, retard de débourrement des bourgeons latéraux ; débourrement anticipé des bourgeons terminaux ; inhibition du débourrement des bourgeons latéraux (effet de dominance apical) ; défaut de couverture foliaire ; forte consommation de réserve au début de la croissance ; développement terminal vigoureux et long (nécessité d'une taille plus sévère) ; formation d'un nombre peu élevé de lambourdes ; retard de l'initiation de la fructification ; fruits petits ; rendement faible ; développement végétatif exubérant ; branches vigoureuses demandant une quantité de froid élevée.

Plusieurs pays ont entrepris des travaux pour mettre au point des techniques permettant d'atténuer les effets de la dormance prolongée. Les recherches ont porté sur l'obtention de variétés à faibles besoins en froid et l'application de techniques culturales ou de produits chimiques susceptibles de stimuler la levée de dormance.
Certaines techniques culturales, notamment la taille, semblent influencer indirectement la satisfaction des besoins en froid de certains cultivars. Leurs effets dépendent de l'intensité de la dormance [2]. La période de taille des arbres influence la durée de la dormance et de floraison. Selon Chandler et Brown [3], une taille sévère effectuée en été retarderait la floraison, alors qu'une taille des arbres ayant déjà reçu au moins 1 mois de froid réduirait la durée de la dormance et avancerait la floraison.

Un grand nombre de produits chimiques de nature très différente ont été testés quant à leur influence sur la levée de dormance des arbres fruitiers. Certains produits se sont révélés aptes à lever la dormance quand le froid est insuffisant : dinitro-orthocrésol (DNOC), nitrate de potassium $\left(\mathrm{KNO}_{3}\right)$, thiourée, benzyladénine (BA), cyanamide d'hydrogène, cytokinines, éthrel, gibbéréllines [4]. Plusieurs de ces produits semblent agir par l'inhibition de la catalase et, par conséquent, ils interviennent à travers l'activation de l'action des péroxydases [5]. D'autres agissent sur la respiration en aérobiose et influencent fortement les bourgeons dormants. Généralement, l'efficacité de tels traitements chimiques dépend de leurs dose et date d'application. Des risques de phytotoxicité sont à craindre surtout sur les organes floraux. À cet égard, les espèces à noyau sont beaucoup plus sensibles que les espèces à pépins dont le bourgeon floral est très protégé [6].

Dans les régions à hivers doux, le traitement des arbres fruitiers par des émulsions d'huiles minérales est le traitement qui a été le plus longtemps utilisé pour lever le repos végétatif. L'époque des pulvérisations d'huile additionnée de DNOC sur les arbres peut avoir des conséquences importantes [7] : les traitements précoces entraînent un forçage de l'arbre qui aboutit à la formation précoce de feuilles et de fleurs sans que soit éliminée pour autant l'irrégularité du débourrement des bourgeons; en revanche, les traitements tardifs permettent de regrouper et d'augmenter le nombre de bourgeons qui s'épanouissent.

Chez la variété 'Golden Delicious', l'utilisation de concentrations élevées d'huile 
$(2,4$ à $8,8 \%)$ à faibles teneurs en DNOC $(0,12 \%)$ a permis d'obtenir le meilleur taux d'ouverture des bourgeons végétatifs latéraux [8]. Dans la région du Saiss au Maroc, la pulvérisation du mélange d'huile de pétrole et de DNOC sur cette même variété a provoqué une augmentation du taux de débourrement, regroupé la floraison et la feuillaison, amélioré la croissance végétative et la qualité du fruit [9]. Dans la région du Gharb (Maroc), l'utilisation d'un mélange à $3 \%$ ou $5 \%$ d'huile de pétrole et à $0,15 \%$ de DNOC a amélioré les taux de débourrement et de floraison et en a avancé les dates. De même, les taux de nouaison, le calibre, le poids moyen de fruit et le rendement ont été augmentés [10]. L'amélioration du débourrement, induite par ces produits, s'est révélée être plus importante pour les bourgeons à bois que pour des bourgeons floraux $[9,10]$.

Le manque en froid constituant un facteur limitant à la production de pommes en zone à hiver doux au Maroc, l'étude menée a eu pour objectif d'évaluer les effets de l'utilisation du DNOC additionné à de l'huile de pétrole sur le débourrement et la production de la variété de pommier 'Golden Delicious' dans les conditions climatiques de Meknès, principale zone de production de pommes au Maroc. Ces travaux ont cherché notamment à confirmer des résultats encourageants obtenus lors d'une première expérimentation conduite sur la saison 1984-1985 [9]. Ils s'inscrivent dans le cadre d'un programme de recherche sur l'utilisation des régulateurs de croissance en arboriculture fruitière, débuté par le département d'Horticulture de l'IAV Hassan II dans les années 80. La confirmation des résultats précédemment obtenus, vérifiés alors dans d'autres conditions climatiques et avec des conduites culturales différentes, devrait conduire à recommander l'utilisation de la technique à l'échelle commerciale.

\section{Matériel et méthodes}

\subsection{Caractéristiques du verger}

L'expérimentation a été menée dans l'unité de production UP 5105 de la Société de
Développement Agricole ( SO.DE.A) située à Boufekrane à une trentaine de kilomètres de Meknès au Maroc. Le sol de cette parcelle d'étude est argilo-calcaire, moyennement profond et de $\mathrm{pH}$ légèrement alcalin.

Alors que l'étude menée pendant la campagne 1984-1985 avait été conduite sur un verger de pommiers 'Golden Delicious' mis en place en 1964, à la densité de plantation de $6 \mathrm{~m} \times 6 \mathrm{~m}$ (277 arbres par ha) et greffés sur pommier franc, l'essai étudié dans le présent travail a été mené sur un verger de la même variété de pommiers, mais greffés sur MM 106 et plantés en 1977 à une distance de $5 \mathrm{~m} \times 2 \mathrm{~m}$, soit une densité de 1000 arbres à l'hectare. Les lignes ont été orientées nord-sud, et les arbres conduits en gobelet libre. Les variétés pollinisatrices, 'Top Red', 'Granny Smith', et 'Ozark Gold' ont été associées à la variété de fond.

\subsection{Traitements appliqués}

L'expérimentation réalisée a cherché à tester l'effet du DNOC, mélangé à deux concentrations différentes d'huile minérale, vis-à-vis de son effet sur la levée de dormance. Les produits alors utilisés ont été :

- la Véraline 3 (Rhône-Poulenc Agro) à $10 \%$ de DNOC en émulsion dans l'huile d'anthracène à $45 \%$;

- l'Oleo 5 (Bayer S.A.) qui contient du parathion à $5 \%$ de matière active en émulsion dans de l'huile à $80 \%$.

Le dispositif adopté a été un bloc aléatoire complet composé de 4 blocs (ou répétitions). Deux facteurs ont été étudiés :

- la date du traitement : le 29 février et le 8 mars 1996 ;

- la dose d'huile utilisée en mélange avec le DNOC : $0 \%$ (témoin), [0,15\% de DNOC + $3 \%$ d'huile de pétrole] et $[0,15 \%$ de DNOC + $5 \%$ d'huile de pétrole].

À chaque date de traitement, les trois modalités du facteur dose ont été appliquées. Six traitements distincts ayant été répétés chacun sur quatre arbres, l'expérimentation a donc porté sur 24 arbres du verger considéré.

Tous les traitements ont été appliqués à l'aide d'un pulvérisateur à dos de 20 L La 
pulvérisation a été réalisée de façon à mouiller uniformémentle bois. Chaque arbre a reçu $4 \mathrm{~L}$ de solution.

\subsection{Observations et mensurations}

\subsubsection{Débourrement et floraison}

L'effet des substances appliquées a été évalué sur le comportement de deux types de rameaux d'âges différents : rameaux de 1 an (notés $R_{1}$ ) et rameaux de 2 ans (notés $R_{2}$ ). Pour chaque traitement, quatre rameaux de chacun de ces deux types, choisis à hauteur d'homme et situés aux quatre points cardinaux de l'arbre pour tenir compte d'un éventuel effet «exposition », ont été marqués.

Sur ces rameaux, un comptage systématique hebdomadaire des bourgeons débourrés, effectué à partir du 2 avril 1996, a permis de contrôler le débourrement. Les stades repères choisis ont été le stade $« \mathrm{C} »$ de Fleckinger pour les bourgeons à fleurs et le stade «début d'étalement des feuilles "pour les bourgeons à bois. Par ailleurs, tout bourgeon floral ayant atteint le stade $\mathrm{F}_{2}$ de Fleckinger a été considéré comme fleuri.

Les taux de débourrement et de floraison ont été calculés par les formules :

- taux de débourrement $(\%)=$ nombre de bourgeons débourrés/nombre total de bourgeons) $\times 100$;

- taux de floraison $(\%)=$ (nombre de bourgeons fleuris/nombre total de bourgeons floraux ) $\times 100$.

\subsubsection{Croissance végétative}

Pour suivre la croissance végétative des rameaux soumis aux différents traitements appliqués, des pousses terminales ont été marquées autour de l'arbre, à hauteur d'homme.

Les mesures de la longueur ont été réalisées une fois tous les 15 j à partir du 20 mai 1996 jusqu'à la fin du mois de juillet.

La vitesse de croissance des pousses a été évaluée par le rapport [accroissement de la longueur/accroissement de temps].

\subsubsection{Nouaison}

Le nombre de fleurs nouées a été compté sur les rameaux $R_{1}$ et $R_{2}$ en prenant comme repère le stade I de Fleckinger. Le taux de nouaison a été évalué par le rapport [(nombre de fleurs nouées / nombre total de fleurs sur le rameau) $\times 100]$.

\subsubsection{Production}

Le nombre de fruits par arbre et leurs poids ont été déterminés à la récolte.

\subsubsection{Analyse statistique des données}

Les mesures ont été transformées en pourcentage et en proportion. Les résultats exprimés sous forme de proportion ont été analysés après leur transformation angulaire $\left(\operatorname{arc} \sin \mathrm{x}^{1 / 2}\right)$ afin de stabiliser les variances.

Les données ont été soumises à une analyse de la variance réalisée au moyen du logiciel Statitcf. La séparation des moyennes a été réalisée à l'aide du test de NewmanKeuls.

\section{Résultats et discussion}

\subsection{Débourrement}

\subsubsection{Bourgeons végétatifs}

\subsubsection{Sur rameaux de 1 an}

Quel que soit le traitement appliqué, le taux de débourrement des bourgeons végétatifs sur rameaux $R_{1}$ a été amélioré durant toute la période de débourrement par rapport aux rameaux témoins non traités (tableau I).

Les observations faites le 2 avril ont montré que ce taux de débourrement différait significativement selon la date à laquelle le mélange [huile + DNOC] avait été appliqué. Cependant, quelle que soit cette date, aucune différence significative n'a été obtenue en fonction des doses $3 \%$ et $5 \%$ d'huile.

À partir des observations faites le 9 avril, les taux de débourrement des bourgeons végétatifs ont varié significativement en fonction des doses d'huile utilisées et, cela, 
Tableau I.

Effet du dinitro-orthocrésol (DNOC) à $0,15 \%$, additionné soit de $3 \%$, soit de $5 \%$ d'huile de pétrole, sur l'évolution du taux de débourrement des bourgeons végétatifs sur rameaux de 1 an $\left(R_{1}\right)$ ou 2 ans $\left(R_{2}\right)$, chez le pommier 'Golden Delicious' cultivé en conditions climatiques de plaine au Maroc.

\begin{tabular}{|c|c|c|c|c|c|c|c|c|c|c|c|c|c|c|c|c|}
\hline \multirow{3}{*}{$\begin{array}{l}\text { Date du } \\
\text { traitement } \\
\text { (1996) }\end{array}$} & \multirow{3}{*}{$\begin{array}{l}\text { Huile } \\
(\%)\end{array}$} & \multicolumn{15}{|c|}{ Taux de débourrement (\%) des bourgeons végétatifs } \\
\hline & & \multicolumn{3}{|c|}{2 avril } & \multicolumn{3}{|c|}{9 avril } & \multicolumn{3}{|c|}{15 avril } & \multicolumn{3}{|c|}{22 avril } & \multicolumn{3}{|c|}{2 mai } \\
\hline & & $\mathrm{R}_{1}$ & $\mathrm{R}_{2}$ & $R_{1}+R_{2}$ & $R_{1}$ & $\mathrm{R}_{2}$ & $R_{1}+R_{2}$ & $R_{1}$ & $\mathrm{R}_{2}$ & $R_{1}+R_{2}$ & $\mathrm{R}_{1}$ & $\mathrm{R}_{2}$ & $R_{1}+R_{2}$ & $R_{1}$ & $\mathrm{R}_{2}$ & $R_{1}+R_{2}$ \\
\hline Témoin & 0 & $0 \mathrm{~b}$ & $0 \mathrm{~b}$ & $0 \mathrm{~b}$ & $1 \mathrm{c}$ & $5 b$ & $3 b$ & $10 \mathrm{c}$ & $11 d$ & $10 \mathrm{~b}$ & $14 \mathrm{c}$ & $15 \mathrm{c}$ & $15 b$ & $18 c$ & $20 \mathrm{c}$ & $19 b$ \\
\hline \multirow[t]{2}{*}{29 février } & 3 & $10 \mathrm{a}$ & $14 \mathrm{a}$ & $12 \mathrm{a}$ & $15 b$ & $21 \mathrm{a}$ & $18 \mathrm{a}$ & $18 \mathrm{bc}$ & $25 \mathrm{bc}$ & $22 \mathrm{a}$ & $22 \mathrm{bc}$ & $32 a b$ & $27 \mathrm{a}$ & $24 \mathrm{bc}$ & $33 a b$ & 29 a \\
\hline & 5 & $21 \mathrm{a}$ & $13 a$ & $17 \mathrm{a}$ & $26 \mathrm{a}$ & $19 a$ & $22 \mathrm{a}$ & $31 \mathrm{a}$ & $21 c$ & $26 \mathrm{a}$ & $34 \mathrm{a}$ & $25 \mathrm{~b}$ & $29 a$ & $36 \mathrm{a}$ & $30 \mathrm{~b}$ & $33 a$ \\
\hline \multirow[t]{2}{*}{08 mars } & 3 & $0 \mathrm{~b}$ & $0 \mathrm{~b}$ & $1 \mathrm{~b}$ & $10 \mathrm{~b}$ & $20 \mathrm{a}$ & $15 \mathrm{a}$ & $19 b$ & $32 a b$ & $26 \mathrm{a}$ & $24 \mathrm{~b}$ & $34 \mathrm{a}$ & $29 a$ & $26 \mathrm{~b}$ & $35 a b$ & $30 \mathrm{a}$ \\
\hline & 5 & $0 \mathrm{~b}$ & $0 \mathrm{~b}$ & $0 \mathrm{~b}$ & $18 a b$ & $25 a$ & $22 \mathrm{a}$ & $22 a b$ & $35 a$ & $28 \mathrm{a}$ & $27 \mathrm{ab}$ & $37 a$ & $32 \mathrm{a}$ & $31 a b$ & $40 \mathrm{a}$ & $35 a$ \\
\hline
\end{tabular}

Dans une même colonne, les moyennes suivies par une lettre différente sont significativement différentes selon le test de NewmanKeuls (seuil de $5 \%$ ).

quelle que soit la date d'application du traitement. Le traitement du 29 février, effectué avec une solution à $0,15 \%$ de DNOC et $5 \%$ d’huile de pétrole, a généré globalement le taux de débourrement le plus important. L'amélioration apportée parl'application de produits chimiques a varié entre $33 \%$ et $100 \%$ par rapport au témoin.

\subsubsection{Sur rameaux de 2 ans}

Tout comme pour les rameaux $R_{1}$, quel que soit le traitement appliqué, le taux de débourrement des bourgeons végétatifs sur rameaux $R_{2}$ a été amélioré durant toute la période de débourrement par rapport aux rameaux témoins non traités (tableau I).

Au début de débourrement, le 2 avril, les taux de débourrement ont significativement différé selon la date d'application du traitement. Toutefois, cette différence s'est estompée avec l'évolution de la campagne. Ainsi, à partir du 9 avril, l'analyse a révélé que les deux concentrations du produit n'entraînaient pas de différence significative des taux de débourrement des bourgeons végétatifs qui variaient cependant de façon significative selon que les arbres avaient été traités ou non. L'amélioration du débourrement apportée par les traitements a varié entre $50 \%$ et $100 \%$ par rapport au témoin.

\subsubsection{Sur rameaux de 1 et 2 ans}

Quelle que soit la date d'observation, le DNOC ajouté à différentes concentrations d'huile a amélioré le débourrement moyen des bourgeons végétatifs sur l'ensemble des rameaux $R_{1}$ et $R_{2}$, par rapport aux rameaux non traités (tableau I). Les solutions appliquées ont donc agi à la fois sur la précocité et sur le regroupement du débourrement. Globalement, il n'est pas apparu d'interactions entre la date d'application et la dose d'huile minérale utilisée, de même il n'y a pas eu non plus d'effet dose ou d'effet date. Toutefois, lors de la première date d'observation, le 2 avril, la date d'application a eu un effet significatif : les traitements précoces ont provoqué un début de débourrement des bourgeons végétatifs, non observé alors ni sur les rameaux traités tardivement, ni sur les rameaux témoins non traités. L'amélioration du débourrement global des bourgeons végétatifs imputable à l'utilisation du mélange [DNOC + huile] a varié de $47 \%$ à $84 \%$. Cependant, il apparaît que les rameaux de 2 ans répondent mieux à l'application de ces produits que les rameaux de 1 an. Les substances utilisées favoriseraient donc mieux le débourrement des bourgeons végétatifs sur vieux bois que sur bois jeune. 


\subsubsection{Bourgeons floraux}

\subsubsection{Sur rameaux de 1 an}

Quelle que soit la date d'application, l'application d'un mélange [DNOC + huile] a amélioré de manière significative le débourrement des bourgeons floraux sur rameaux $\mathrm{R}_{1}$ par rapport aux rameaux témoins non traités (tableau II).

Le traitement précoce du 29 février à $5 \%$ d'huile minérale a induit un taux de débourrement des bourgeons floraux légèrement supérieur à celui des autres traitements. Toutefois, globalement, le taux de débourrement final observé le 2 mai a été le même pour tous les arbres traités. L'amélioration du débourrement des bourgeons floraux sur rameaux de 1 an imputable aux solutions utilisées a varié de $25 \%$ à $46 \%$ par rapport au témoin.

\subsubsection{Sur rameaux de 2 ans}

Quelle que soit la date d'observation considérée, la solution appliquée sur les rameaux de 2 ans a amélioré le débourrement des bourgeons floraux (tableau II).

L'effet date ne s'est manifesté qu'au début de la phase de débourrement et s'est estompé par la suite. Il n'est pas apparu de différences significatives en fonction des deux concentrations d'huile minérale utilisées.

L'ensemble des arbres traités a eu un taux de débourrement similaire et très nettement supérieur à celui enregistré sur les arbres témoins. L'amélioration apportée par ces traitements a varié entre $51 \%$ et $76 \%$.

\subsubsection{Sur rameaux de 1 et 2 ans}

Globalement, le débourrement des bourgeons floraux sur l'ensemble des rameaux de 1 an et de 2 ans a été amélioré de manière significative par l'utilisation des substances chimiques (tableau II). À l'exception de la première date d'observation du 2 avril, il n'y a eu ni effet date d'application, ni effet concentration du produit utilisé. En revanche, les arbres traités ont présenté des taux de débourrement de bourgeons floraux nettement supérieurs à ceux enregistrés sur les arbres témoins. L'amélioration du débourrement global des bourgeons floraux imputable à l'utilisation du mélange [DNOC + huile] a varié de $46 \%$ à $59 \%$ par rapport au témoin ; elle a été plus importante sur rameaux de 2 ans que sur ceux de 1 an. En effet, dans des conditions de froid hivernal insuffisant, la baisse de débourrement enregistrée est attribuée à un faible débourrement sur vieux bois. Les substances chimiques permettent ainsi d'améliorer le

\section{Tableau II.}

Effet du dinitro-orthocrésol (DNOC) à 0,15\%, additionné soit de $3 \%$, soit de $5 \%$ d'huile de pétrole, sur l'évolution du taux de débourrement des bourgeons floraux sur rameaux de 1 an $\left(R_{1}\right)$ ou 2 ans $\left(R_{2}\right)$, chez le pommier 'Golden Delicious' cultivé en conditions climatiques de plaine au Maroc.

\begin{tabular}{|c|c|c|c|c|c|c|c|c|c|c|c|c|c|c|c|c|}
\hline \multirow{3}{*}{$\begin{array}{l}\text { Date du } \\
\text { traitement } \\
\text { (1996) }\end{array}$} & \multirow{3}{*}{$\begin{array}{l}\text { Huile } \\
(\%)\end{array}$} & \multicolumn{15}{|c|}{ Taux de débourrement (\%) des bourgeons floraux } \\
\hline & & \multicolumn{3}{|c|}{2 avril } & \multicolumn{3}{|c|}{9 avril } & \multicolumn{3}{|c|}{15 avril } & \multicolumn{3}{|c|}{22 avril } & \multicolumn{3}{|c|}{2 mai } \\
\hline & & $\mathrm{R}_{1}$ & $\mathrm{R}_{2}$ & $R_{1}+R_{2}$ & $\mathrm{R}_{1}$ & $\mathrm{R}_{2}$ & $R_{1}+R_{2}$ & $\mathrm{R}_{1}$ & $\mathrm{R}_{2}$ & $\mathrm{R}_{1}+\mathrm{R}_{2}$ & $\mathrm{R}_{1}$ & $\mathrm{R}_{2}$ & $\mathrm{R}_{1}+\mathrm{R}_{2}$ & $\mathrm{R}_{1}$ & $\mathrm{R}_{2}$ & $R_{1}+R_{2}$ \\
\hline Témoin & 0 & $0 \mathrm{~b}$ & $0 \mathrm{~b}$ & $\mathrm{Ob}$ & $24 c$ & $10 \mathrm{c}$ & $17 \mathrm{~b}$ & $32 d$ & $28 \mathrm{~b}$ & $30 \mathrm{~b}$ & $44 \mathrm{c}$ & $36 \mathrm{~b}$ & $40 \mathrm{~b}$ & $48 \mathrm{~b}$ & $45 \mathrm{~b}$ & $46 \mathrm{~b}$ \\
\hline \multirow[t]{2}{*}{29 février } & 3 & $24 \mathrm{a}$ & $11 a b$ & $17 \mathrm{a}$ & $37 \mathrm{~b}$ & $32 \mathrm{~b}$ & $34 \mathrm{a}$ & $51 \mathrm{ab}$ & $44 \mathrm{a}$ & $47 \mathrm{a}$ & $60 a b$ & $53 \mathrm{a}$ & $56 \mathrm{a}$ & $65 \mathrm{a}$ & $69 \mathrm{a}$ & 67 a \\
\hline & 5 & $27 \mathrm{a}$ & $18 \mathrm{a}$ & $22 \mathrm{a}$ & $50 a$ & $43 a$ & $46 \mathrm{a}$ & $56 \mathrm{a}$ & $47 a$ & $52 \mathrm{a}$ & $64 \mathrm{a}$ & $55 a$ & $59 a$ & $70 \mathrm{a}$ & $76 \mathrm{a}$ & $73 a$ \\
\hline \multirow[t]{2}{*}{08 mars } & 3 & $2 b$ & $7 \mathrm{~b}$ & $4 \mathrm{~b}$ & $32 \mathrm{~b}$ & $36 a b$ & $34 \mathrm{a}$ & $41 \mathrm{c}$ & $48 \mathrm{a}$ & $45 a$ & $52 \mathrm{~b}$ & $63 a$ & $57 a$ & $60 \mathrm{a}$ & $79 a$ & $70 \mathrm{a}$ \\
\hline & 5 & $2 \mathrm{~b}$ & $3 \mathrm{~b}$ & $3 \mathrm{~b}$ & $37 \mathrm{~b}$ & $35 \mathrm{ab}$ & $36 \mathrm{a}$ & $45 \mathrm{bc}$ & $40 a$ & $42 a$ & $56 a b$ & $56 a$ & $56 a$ & $60 \mathrm{a}$ & $68 \mathrm{a}$ & $64 \mathrm{a}$ \\
\hline
\end{tabular}

Dans une même colonne, les moyennes suivies par une lettre différente sont significativement différentes selon le test de Newman-Keuls (seuil de $5 \%$ ). 
débourrement global de l'arbre en forçant les bourgeons qui seraient restés latents à démarrer.

\subsubsection{Précocité du débourrement}

La précocité du débourrement a été évaluée en comparant la date à laquelle les rameaux témoins non traités ont atteint leur taux de débourrement maximal pour chaque type de bourgeon (végétatif et floral) avec les dates respectives auxquelles les arbres traités ont atteint le même niveau de débourrement.

L'application des substances chimiques utilisées a permis d'avancer la date de débourrement aussi bien pour les bourgeons végétatifs que pour les bourgeons floraux. Cet avancement a varié de $22 \mathrm{j}$ à $25 \mathrm{j}$ pour les bourgeons végétatifs et de $16 \mathrm{j}$ à $23 \mathrm{j}$ pour les bourgeons floraux. L'effet des substances chimiques se confirme donc être plus marqué sur bourgeons végétatifs que sur bourgeons floraux. La précocité du débourrement et l'amélioration de son taux rapportée précédemment permettraient une formation rapide du fruit et le développement d'une feuillaison adéquate susceptible d'assurer une production normale de pommes d'un calibre commercialisable.

\subsection{Floraison}

\subsection{1. Évolution du taux de floraison}

L'application du DNOC additionné d'huile du pétrole a permis, en plus de l'amélioration du taux de débourrement des bourgeons floraux, d'augmenter le taux de bourgeons floraux épanouis (figure 1). Chez les rameaux témoins non traités dont le taux de débourrement était déjà faible (46\%, tableau II), seulement $43 \%$ de ces bourgeons ont évolué en fleurs (figure 1). En absence de traitement, il y aurait donc avortement d'une proportion importante de bourgeons floraux. Ce désordre physiologique est connu pour être l'un des symptômes caractéristiques d'une insuffisance en froid. En contrepartie, le taux de floraison des arbres traités a varié de $80 \%$ à $97 \%$, ce qui constitue une amélioration très importante par rapport au témoin. Par ailleurs, la floraison a été beaucoup plus étalée sur les arbres témoins que sur les arbres traités, chez lesquels un groupement de la floraison permettrait de réunir des conditions favorables à une bonne nouaison propice à la formation de fruits capables de poursuivre leur développement jusqu’à maturité.

\subsubsection{Précocité de floraison}

La date de pleine floraison a été avancée par les traitements chimiques (figure 1). Cet avancement a varié entre $6 \mathrm{j}$ et $9 \mathrm{j}$. L'application de DNOC additionné d'huile de pétrole a donc agi sur la période de floraison qui a eu lieu précocement par rapport au témoin, et ce quelle que soient la date et la dose d'application.

\subsection{Nouaison}

Le taux de nouaison n'a pas été affecté par les substances chimiques. Il n'y a eu ni effet date, ni effet dose, ni interaction entre les deux facteurs. Le DNOC additionné d'huile du pétrole semblerait donc ne pas influencer le taux de nouaison. Malgré la grande différence existant entre les taux de floraison des arbres traités et ceux des témoins, les taux de nouaison sont restés similaires et compris entre $22 \%$ et $27 \%$.

\subsection{Croissance végétative}

Le DNOC en émulsion dans l'huile minérale a permis une plus forte croissance des pousses terminales, reflétant ainsi son effet

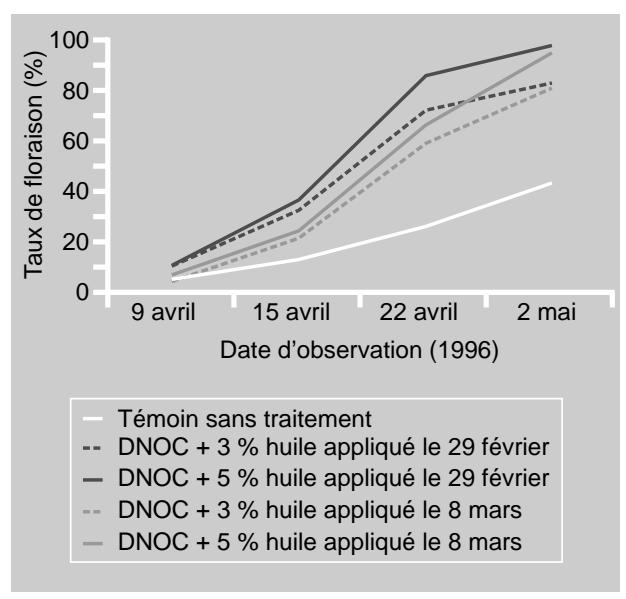

Figure 1.

Effets du dinitro-orthocrésol (DNOC) à $0,15 \%$, additionné d'huile de pétrole, et de la date d'application du traitement, sur le taux de floraison du pommier 'Golden Delicious' dans les conditions climatiques de plaine au Maroc. 
Figure 2.

Effets de dinitro-orthocrésol (DNOC) à $0,15 \%$, additionné d'huile de pétrole, et de la date d'application du traitement, sur la croissance végétative du pommier 'Golden Delicious' dans les conditions climatiques de plaine au Maroc. La croissance végétative est évaluée par la mesure de la longueur de la pousse terminale.

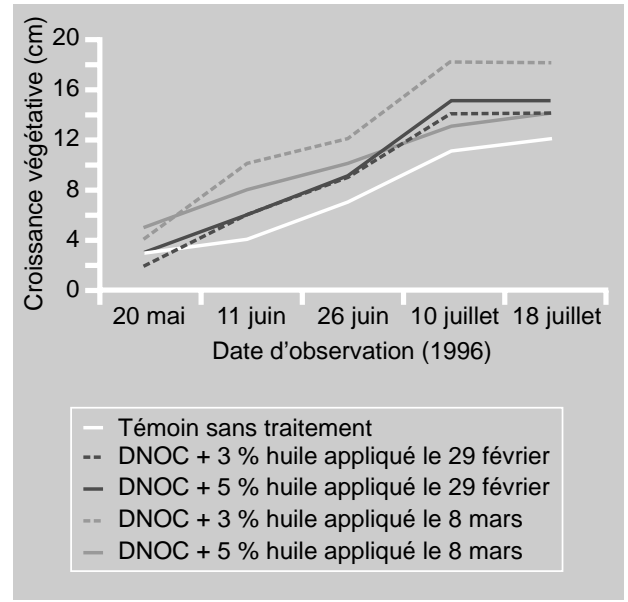

sur la vitesse de croissance de ces rameaux (figure 2). Le traitement tardif du 8 mars à $0,15 \%$ DNOC et $3 \%$ d'huile a donné l'allongement le plus élevé $(18 \mathrm{~cm})$. Pour ce traitement, la vitesse d'allongement des pousses a été la plus élevée entre le 26 juin et le 10 juillet. Ainsi, l'effet positif du DNOC sur le taux de débourrement des bourgeons végétatifs et floraux et sur leur précocité s'est également répercuté sur la croissance végétative des rameaux.

\subsection{Nombre de fruits et rendement}

Le DNOC additionné d'huile du pétrole a augmenté de façon significative le nombre de fruits par arbre pour tous les traitements (tableau III).
Les arbres traités ont présenté un nombre de fruits, un poids de fruits et un rendement par arbre supérieurs à ceux du témoin (tableau III). L'application tardive du 8 mars d'une solution de $0,15 \%$ de DNOC ajouté à $5 \%$ d'huile a donné les meilleurs résultats. Le rendement imputable à ce traitement a permis de dépasser celui du témoin de $112,5 \%$, alors que l'amélioration due aux autres traitements a varié de $25 \%$ à $56 \%$ par rapport au témoin. Cet accroissement du rendement a résulté de l'augmentation du nombre de fruits par arbre et de l'amélioration du calibre et du poids du fruit. Ainsi, l'utilisation du DNOC mélangé à de l'huile minérale a permis d'améliorer le rendement en pommes sur les plans quantitatif et qualitatif, ce qui est l'objectif généralement recherché par les arboriculteurs.

\section{Discussion et conclusion}

Le DNOC additionné d'huile du pétrole a amélioré de manière significative le taux de débourrement. L'amélioration induite par les traitements a été beaucoup plus importante sur le bois de 2 ans que sur le bois de 1 an, quel que soit le type de bourgeon considéré. Le taux de débourrement des bourgeons végétatifs a été supérieur à celui des bourgeons floraux, quel que soit l'âge du bois. Les effets du DNOC additionné

Tableau III.

Effet du dinitro-orthocrésol (DNOC) à $0,15 \%$, additionné soit de $3 \%$, soit de $5 \%$ d'huile de pétrole, sur le rendement, le nombre de fruit par arbre et le poids du fruit chez 'Golden Delicious' dans les conditions climatiques de plaine au Maroc.

\begin{tabular}{|c|c|c|c|c|}
\hline Date (1996) & Huile (\%) & Rendement $\left(\mathrm{t} \times \mathrm{ha}^{-1}\right)$ & Nombre de fruits par arbre & Poids du fruit (g) \\
\hline Témoin & 0 & $16 d$ & $180 \mathrm{~d}$ & $88 c$ \\
\hline \multirow[t]{2}{*}{29 février } & 3 & $20 \mathrm{c}$ & $197 \mathrm{c}$ & $102 \mathrm{~b}$ \\
\hline & 5 & $25 \mathrm{~b}$ & $226 \mathrm{~b}$ & $111 \mathrm{~b}$ \\
\hline \multirow[t]{2}{*}{8 mars } & 3 & $23 b$ & $221 b$ & $104 \mathrm{~b}$ \\
\hline & 5 & $34 \mathrm{a}$ & 237 a & $143 \mathrm{a}$ \\
\hline
\end{tabular}


d'huile du pétrole se sont donc davantage manifestés sur bois âgé et sur bourgeons végétatifs que sur bois jeune et sur bourgeons floraux. Cela permettrait d'atténuer les désordres physiologiques observés chez le pommier 'Golden Delicious' dans des conditions d'insuffisance en froid hivernal, puisque, dans les régions à hiver doux, les problèmes de débourrement touchent principalement les bourgeons présents sur bois âgé et s'expriment par une insuffisance de feuillaison qui seraitle résultat d'un manque de débourrement des bourgeons végétatifs.

Comme dans le cas des résultats présentés à l'issue de ces travaux portant sur la période 1995-1996, l'expérimentation de 1984-1985 avait montré que le DNOC additionné d'huile du pétrole améliorait le débourrement des bourgeons végétatifs et floraux sur les deux types de rameaux âgés de 1 et de 2 ans [9]. Par ailleurs, lors de cette campagne, les traitements avaient amélioré le débourrement des bourgeons végétatifs sur rameaux de 1 an de $32 \%$ à $110 \%$ et ceux sur rameaux de 2 ans, de $80 \%$ à $87 \%$, par rapport au témoin. Parallèlement, l'amélioration induite sur le débourrement des bourgeons floraux avait varié de $7 \%$ à $39 \%$ sur rameaux de 1 an et de $16 \%$ à $25 \%$ sur rameaux de 2 ans. Globalement, l'amélioration du taux de débourrement enregistrée sur l'ensemble des rameaux avait varié entre $53 \%$ et $106 \%$ pour les bourgeons végétatifs et entre $13 \%$ et $30 \%$ pour les bourgeons floraux. De plus, lors de cette campagne 1984-1985, le DNOC avait induit une précocité du débourrement des bourgeons de l'ordre de 2 semaines, quelque peu inférieure aux 3 semaines observées lors de la dernière étude. Le débourrement précoce s'était répercuté sur la date de floraison qui avait été avancée à son tour et la proportion des bourgeons floraux atteignant la pleine floraison avait également été augmentée.

Dans les deux expérimentations, le DNOC a donc eu un effet forçant supérieur sur vieux bois que sur bois jeune et sur bourgeons végétatifs que sur bourgeons floraux, ainsi qu'un effet d'avancement, d'augmentation et de regroupement de la floraison. Ces améliorations se sont répercutées sur la production surtout influencée par une application tardive du DNOC à 0,15\% additionné à $5 \%$ d'huile. L'utilisation de ce mélange a donc permis de remédier aux anomalies observées chez le pommier souffrant d'un manque de froid. Elle peut donc être recommandée aux arboriculteurs de la région de Meknès au Maroc pour pallier les problèmes de froid hivernal observés en cet endroit.

\section{Références}

[1] Petri J.L., L'interruption de dormance de pommier, BASF informations agricoles 2 (1989) 17-20.

[2] Walker D.R., Seeley S.D., The rest mechanism in deciduous fruit trees as influenced by plant growth substances, Acta Hortic. 34 (1973) 235-239.

[3] Chandler W.H., Brown D.S., Deciduous orchards in California winters, Calif. Agr. Exp. Serv. Circular, 179, 1951.

[4] Erez A., Lavee S., The effect of climatic conditions on dormancy development of peach buds, J. Am. Soc. Hortic. Sci. 96 (1971) 711-714.

[5] Taylorson R.B., Hendricks S.B., Dormancy in seed, Annu. Rev. Plant Phys. 28 (1977) 331-354.

[6] Erez A., Chemical control of bud break, HortScience 22 (1987) 1240-1243.

[7] Diaz D.H., Alvarez A., Sandoval J., Cultural and chemical practices to induce uniform bud break of peach and apple under warm climates in Mexico, Acta Hortic. 199 (1987) 99-136.

[8] Erez A., Zur A., Breaking the rest of apple buds by narrow-distillation-range oil and dinitro-o-cresol, Scientia Hortic. 14 (1981) 47-54.

[9] Mahhou A.,Taoura M., Walali Loudiyi D., Effects of dinitro-ortho-cresol (DNOC) and oil on bud break of 'Golden Delicious' apple in the Saiss area of Morocco, Actes Institut Agronomique Vétérinaire (Maroc) 10 (3) (1990) 27-32.

[10] Mahhou A., Hakam M., Effets du mélange dinitro-orthocresol-huile de pétrole sur la levée de dormance du pommier (Malus domestica L.) dans la région du Gharb, Actes Institut Agronomique Vétérinaire (Maroc) 15 (4) (1995) 37-42. 


\section{Efectos del dinitro ortocresol (DNOC) en la ruptura del letargo del manzano 'Golden Delicious' cultivado en Ilanura marroquí.}

Resumen - Introducción. El insuficiente frío invernal en las zonas de llanura de Marruecos constituye un factor limitante para la producción del manzano. Los efectos del dinitro ortocresol (DNOC), producto conocido por romper la latencia en otros árboles frutales, se evaluaron con respecto al desborre de manzanos 'Golden Delicious' cultivados en estas condiciones climáticas. Material y métodos. Una mezcla de DNOC al $0,15 \%$ y de aceite mineral utilizado al $3 \%$ o 5\% se aplicó a los manzanos, en dos fechas diferentes. La producción de los árboles tratados se comparó con la de los testigos no tratados. La evaluación de los tratamientos se efectuó con respecto a las tasas de desborre de yemas vegetativas y florales, floración, fructificación, crecimiento vegetativo y producción. Resultados. La solución utilizada adelantó la fecha de desborre y mejoró notablemente las tasas de desborre de las yemas vegetativas y florales, el crecimiento vegetativo y el rendimiento y calibre de los frutos. Conclusión. La mezcla [DNOC + aceite mineral] permitió adelantar y agrupar el desborre del conjunto de las yemas y mejoró el calibre y la producción del manzano 'Golden Delicious'. Esta mezcla podría, por tanto, utilizarse para paliar la falta de frío invernal en las zonas de llanura marroquíes caracterizadas por sus suaves inviernos.

Marruecos / Malus / salida de la latencia / dnoc / aceite mineral / crecimiento / floración / fructificación / rendimiento 